\title{
Project SANC (former CalcPHEP): Support of Analytic and Numeric calculations for experiments at Colliders
}

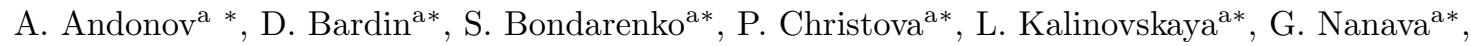 \\ G. Passarino ${ }^{\mathrm{b} \dagger}$

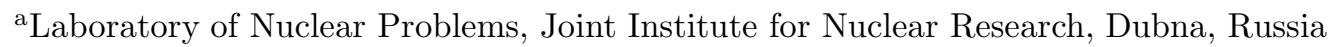 \\ ${ }^{\mathrm{b}}$ Department of Theoretical Physics, University of Torino, INFN, Torino, Italy
}

The project, aimed at the theoretical support of experiments at modern and future accelerators - TEVATRON, LHC, electron Linear Colliders (TESLA, NLC, CLIC) and muon factories, is presented. Within this project a four-level computer system is being created, which must automatically calculate, at the one-loop precision level the pseudo- and realistic observables (decay rates and event distributions) for more and more complicated processes of elementary particle interaction, using the principle of knowledge storing.

It was already used for a recalculation of the EW radiative corrections for Atomic Parity Violation [1] and complete one-loop corrections for the process $e^{+} e^{-} \rightarrow t \bar{t}$ [2, 3, 4]; for the latter an, agreement up to 11 digits with FeynArts and the other results is found. The version of SANC that we describe here is capable of automatically computing the decay rates and the distributions for the decays $Z(H, W) \rightarrow f \bar{f}$ in the one-loop approximation.

\section{SANC PROJECT AND ITS ROOTS}

The main goal of this project is the creation of a software product, accessible via Internet, for the automatic computation of pseudo- and realistic observables with a one-loop precision for various processes of elementary particle interactions, such as: $1 \rightarrow 2,1 \rightarrow 3,2 \rightarrow 2,1 \rightarrow 4,2 \rightarrow 3$, etc. [5].

It has two roots: 1) Codes aimed at a theoretical support of HEP experiments, such as TOPAZO [6], ZFITTER [7] and similar ones.

2) Numerous FORM2-codes, written by the authors of ref. [8] while they were working on it.

It is supposed that the main software products of the project should be the Monte-Carlo event generators that are being created in collaboration with S. Jadach and Z. Was from INF (Krakow, Poland) and B.F.L. Ward from University of Tennessee (Knoxville, USA). On top of these generators, for some processes the semi-analytic codes are also provided, the latter being used both for

\footnotetext{
*Supported by INTAS grant $\mathrm{N}^{o} 00-00313$

† Supported by the European Union under contract HPRN-CT-2000-00149 and by MIUR under contract 2001023713006.
}

the cross-checks of MC generators and for fits of inclusive observables to the theory predictions.

\section{BASIC NOTIONS}

\subsection{The SM Lagrangian in the $R_{\xi}$ gauge}

All the calculations start from the SM Lagrangian in the $R_{\xi}$ gauge. It depends on 25 input parameters (IPS), fields, and on three gauge parameters:

$\mathcal{L}=\mathcal{L}$ (IPS: 25 parameters, fields, $\left.\xi_{A}, \xi_{Z}, \xi\right)$,

where the fields are: fermions, vector bosons, physical Higgs field, $H$, and unphysical fields, $\phi^{0}, \phi^{ \pm}, Y^{A}, Y^{Z}, X^{ \pm}[8]$. We give the example of Feynman Rules for vector boson propagators:

$$
\begin{aligned}
& A: \quad \frac{1}{p^{2}}\left\{\delta_{\mu \nu}+\left(\xi_{A}^{2}-1\right) \frac{p_{\mu} p_{\nu}}{p^{2}}\right\}, \\
& Z: \frac{1}{p^{2}+M_{z}^{2}}\left\{\delta_{\mu \nu}+\left(\xi_{z}^{2}-1\right) \frac{p_{\mu} p_{\nu}}{p^{2}+\xi_{z}^{2} M_{z}^{2}}\right\}, \\
& W^{ \pm}: \frac{1}{p^{2}+M_{W}^{2}}\left\{\delta_{\mu \nu}+\left(\xi^{2}-1\right) \frac{p_{\mu} p_{\nu}}{p^{2}+\xi^{2} M_{W}^{2}}\right\} .
\end{aligned}
$$

\subsection{Scalar functions and reduction}

At present, SANC knows how to deal with up to third-rank tensorial reduction to the usual scalar 
functions: $A_{0}, B_{0}, C_{0}$ and $D_{0}$; and to the auxiliary scalar functions: $a_{0}, b_{0}, c_{0}$ and $d_{0}$, which are due to particular form of photonic propagator in $R_{\xi}$ gauge. A new fortran library for numerical calculations of all these functions but one $\left(D_{0}\right)$ is created and thoroughly tested by means of comparison with the other codes. For the $D_{0}$ function, we use an old coding of TOPAZO [6].

\subsection{Amplitude basis, scalar form factors, helicity amplitudes}

SANC computes the one-loop covariant amplitude $(\mathcal{A})$ of a process parametrized in a certain basis by a certain number of scalar form factors (SFF). Next, it computes helicity amplitudes (HA) in terms of SFF. As an example, we present three covariant amplitudes of the decays $B(Q) \rightarrow f\left(p_{1}\right) \bar{f}\left(p_{2}\right)$, where $B=H, Z, W:$

$H \rightarrow f \bar{f}: \mathcal{A} \propto I \mathcal{F}_{S}$,

1 structure (S-basis), $1 \mathrm{SFF}, 1 \mathrm{HA}$;

$Z \rightarrow f \bar{f}: \mathcal{A} \propto i \gamma_{\mu} \gamma_{6} \mathcal{F}_{\mathbf{L}}+i \gamma_{\mu} \mathcal{F}_{\mathbf{Q}}+m_{f} D_{\mu} \mathcal{F}_{\mathbf{D}}$,

3 structures (LQD-basis), $3 \mathrm{SFF}, 3 \mathrm{HA}$;

$$
\begin{aligned}
W \rightarrow u \bar{d}: \mathcal{A} \propto i \gamma_{\mu} \gamma_{6} \mathcal{F}_{\mathbf{L}}+i \gamma_{\mu} \gamma_{7} \mathcal{F}_{\mathbf{R}} & \\
& +m_{u} D_{\mu} \gamma_{6} \mathcal{F}_{\mathbf{L D}}+m_{d} D_{\mu} \gamma_{7} \mathcal{F}_{\mathbf{R D}},
\end{aligned}
$$

4 structures (LRD-basis), $4 \mathrm{SFF}, 4 \mathrm{HA}$,

where $D_{\mu}=\left(p_{1}-p_{2}\right)_{\mu}$. Besides SFF, the HA depend on kinematical factors and coupling constants. For instance, for $Z$ decay three HA are:

$$
\begin{aligned}
& \mathbf{A}_{\mathbf{0} \pm \pm}^{\mathbf{z}}=\frac{g m_{f}}{c_{W}}\left[a_{f} \mathcal{F}_{\mathbf{L}}+\delta_{f} \mathcal{F}_{\mathbf{Q}}+\frac{1}{2} a_{f} \beta_{f}^{2} M_{Z}^{2} \mathcal{F}_{\mathbf{D}}\right], \\
& \mathbf{A}_{ \pm \pm \mp}^{\mathbf{z}}=\frac{g M_{Z}}{\sqrt{2} c_{W}}\left[a_{f}\left(1 \mp \beta_{f}\right) \mathcal{F}_{\mathbf{L}}+\delta_{f} \mathcal{F}_{\mathbf{Q}}\right]
\end{aligned}
$$

where $\beta_{f}^{2}=1-4 \frac{m_{f}^{2}}{M_{z}^{2}}$,

and $\delta_{f}=v_{f}-a_{f}=-2 Q_{f} s_{W}^{2}, \quad a_{f}=I_{f}^{(3)}$.

We note that the number of SFF and the number of independent non-zero HA always coincide. In general, a $2 f \rightarrow 2 f$ process with four different external fermion masses is described by $16 \mathrm{SFF}$ and 16 independent HA. In case of $e^{+} e^{-} \rightarrow t \bar{t}$ process, one has six SFF and six independent HA if the electron mass is ignored [2, 3, 4 ].

\section{STATUS OF THE PROJECT}

\subsection{Four levels of computer system}

Here we explain what the SANC system does at its four levels in the case of calculation of pseudoobservables (decay rates, event distributions) for the simplest decays: $H(Z, W) \rightarrow f_{1} \bar{f}_{2}$.

Level 1: the chain of calculations from $\mathcal{L}_{S M}$ to the ultraviolet-free helicity amplitudes is realized. (All codes are written in FORM3 [9]). It contains four sublevels, which calculate:

- the scalar form factors;

- the soft and hard photonic contributions to the decay rates (analytic chain);

- the helicity amplitudes for basic processes;

- the helicity amplitudes for accompanying bremsstrahlung processes;

Level 2: an s2n.f software generates automatically the fortran codes for $\Gamma^{(1)}=\Gamma^{\text {Born }}+$ $\Gamma^{\text {Virt }}+\Gamma^{\text {Soft }}+\Gamma^{\text {Hard }}$

Level 3: an infrared rearrangement (or exponentiation) procedure should work here (it is still at the stage of development);

Level 4: a Monte Carlo event generator works out. For the time being, we have here a 'manually written' fortran code.

\subsection{Some keywords, characterizing SANC}

SANC is an Internet-based system (address http://brg.jinr.ru (h) the use of which is supposed to be as simple as the usual surf on Internet. It is a database-based system. This means that there is a storage of source codes written in several languages, which talk to one another, being placed into a homogeneous environment written in JAVA (linker). It uses the precomputation or intermediate access principle which means that:

1) All the relevant one-loop diagrams are precomputed and stored. This greatly saves the CPU.

2) It has several intermediate 'entries' to bypass CPU consuming computations. The user may also access its final product, a MC generator. From the other side, the full chain of calculation 'from the Lagrangian to realistic distribution' can be worked out in real time upon the user request. This option is intended to demonstrate the selfreproducibility of the full chain of calculation at any time. 


\section{First versions of SANC}

- v0.01, 3/01 realizes a part of analytic calculations of level 1 for the decays $H$ $(Z, W) \rightarrow f_{1} \bar{f}_{2}$ (demonstration of viability);

- several versions, v0.02c/d, were tested on the road toward realization of levels 2,4 for decays $H(Z, W) \rightarrow f_{1} \bar{f}_{2}$;

- the current version, v0.03 (summer'02), realizes the full chain of calculations, returns numbers and distributions for these decays at the one-loop level of precision (demonstration of workability);

- the next version, v0.10 (at work), should contain all FORM3 codes of level 1 for the processes $2 f \rightarrow 2 f$ and the decays $F \rightarrow 3 f$.

\subsection{Comparison with other codes}

A lot of comparisons with the other codes were made. For instance, we compared numbers with those of the ZFITTER code for all channels of $e^{+} e^{-} \rightarrow f \bar{f}$ with light fermion masses and found the following agreement: within $\mathbf{8 - 9}$ digits for the SFF; within $\mathbf{7 - 8}$ digits for the one-loop differential cross-sections $d \sigma^{(1)} / d \cos \vartheta$; within $6-\mathbf{7}$ digits for the total cross section and $\sigma^{F B}$.

Several comparisons were made for the $e^{+} e^{-} \rightarrow$ $t \bar{t}$ process. First is an internal one, between two fortran codes: s2n.f and eeffLib. The latter is a 'manually written' code aimed at benchmarking of s2n.f software. For the SFF and for the complete one-loop differential crosssection $d \sigma^{(1)} / d \cos \vartheta$ the numbers agree within 12-13 digits. A comparison between s2n.f and FeynArts 10 for the one-loop cross-section without soft photons revealed an agreement within 11 digits. Finally, we compared s2n.f numbers with those of the topfit code 11,12 for the differential one-loop cross-section with soft photons and found an 8-digit agreement.

\section{CONCLUDING REMARKS}

SANC clearly is a long-term project. Its first phase is realized in demonstration version 0.10.

Its second phase assumes the creation of a complete software product, accessible via an Internetbased environment, and realizing the full chain of calculations at the one-loop level of precision including processes $2 \rightarrow 3$ and decays $1 \rightarrow 4$.

\section{REFERENCES}

1. D. Y. Bardin, P. Christova, L. Kalinovskaya and G. Passarino, Eur. Phys. J. C22 (2001) 99-104.

2. D. Bardin, L. Kalinovskaya and G. Nanava, "An electroweak library for the calculation of EWRC to $e^{+} e^{-} \rightarrow f \bar{f}$ within the CalcPHEP project", CERN-TH/2001-308, hep-ph/0012080.

3. A. Andonov, D. Bardin, S. Bondarenko, P. Christova, L. Kalinovskaya and G. Nanava, "Further study of the $e^{+} e^{-} \rightarrow f \bar{f}$ process with the aid of CalcPHEP system", CERN$\mathrm{TH} / 2002-068$, hep-ph/0202112.

4. A. Andonov, D. Bardin, S. Bondarenko, P. Christova, L. Kalinovskaya and G. Nanava, "Update of one loop corrections for $e^{+} e^{-} \rightarrow f \bar{f}$, first run of CalcPHEP system", hep-ph/0207156.

5. D. Bardin et al., "Project CalcPHEP: Calculus for precision high energy physics", hep-ph/0202004.

6. G. Montagna, O. Nicrosini, F. Piccinini and G. Passarino, Comput. Phys. Commun. 117 (1999) 278.

7. D. Bardin, M. Bilenky, P. Christova, M. Jack, L. Kalinovskaya, A. Olshevsky, S. Riemann and T. Riemann, Comput. Phys. Commun. 133 (2001) 229-395.

8. D. Y. Bardin and G. Passarino, The standard model in the making: Precision study of the electroweak interactions. Oxford, UK: Clarendon (1999) $685 \mathrm{p}$.

9. J. A. M. Vermaseren, "New features of FORM", math-ph/0010025.

10. T. Hahn, Nucl. Phys. Proc. Suppl. 89 (2000) 231-236.

11. J. Fleischer, T. Hahn, W. Hollik, T. Riemann, C. Schappacher and A. Wertenbach, "Complete electroweak one-loop radiative corrections to top-pair production at TESLA: - a comparison", hep-ph/0202109.

12. J. Fleischer, J. Fujimoto, T. Ishikawa, A. Leike, T. Riemann, Y. Shimizu and A. Wertenbach, "One-loop corrections to the process $e^{+} e^{-} \rightarrow t \bar{t}$ including hard bremsstrahlung", hep-ph/0203220. 